Pak. J. Weed Sci. Res., 27(3): 307-320, 2021307

\title{
WEED MANAGEMENT AND YIELD IMPROVEMENT IN MUNGBEAN THROUGH ALLELOPATHIC ACTION OF SUNFLOWER AND BERMUDA GRASS EXTRACTS IN CONJUNCTION WITH IRRIGATION FREQUENCIES
}

\author{
Muhammad Naeem Korejo ${ }^{1 *}$, Muhammad Nawaz Kandhro ${ }^{1}$, Aijaz Ahmed Soomro ${ }^{1}$ and Niaz \\ Ahmed Wahocho ${ }^{2}$
}

\begin{abstract}
A field study was conducted to evaluate the allelopathic potential of sunflower (Helianthus annuus L.) and bermuda grass (Cynodon dactylon L.) extracts under various irrigation levels on weed density and yield of mungbean cultivar AEM-96. The experiment consisted of different weed control practices i.e. weedy check, various levels of sunflower / bermuda grass extracts, herbicides and hand weeding under three irrigation frequencies $(2$, 3 and 4). The analysis variance showed that various weed control practices caused significant suppression of weeds and increased seed yield as compared to weedy check. The application of sunflower extract @ $15 \mathrm{~L} \mathrm{ha}^{-1}$ in combination with herbicides (atlantis @ $0.1 \mathrm{~L}$ $\mathrm{ha}^{-1}$ + puma super @ $0.156 \mathrm{~L} \mathrm{ha}^{-1}$ ) resulted in minimum weed parameters viz. density $(52.0$ $\left.\mathrm{m}^{-2}\right)$ and dry weight $\left(99.0 \mathrm{~g} \mathrm{~m}^{-2}\right)$ and produced maximum mungbean growth and yield traits particularly seed yield (2404 kg ha-1). Bermuda grass extract @ $15 \mathrm{~L} \mathrm{ha}^{-1}$ in integration with herbicides (atlantis @ $0.1 \mathrm{~L} \mathrm{ha}^{-1}+$ puma super @ $0.156 \mathrm{~L} \mathrm{ha}^{-1}$ ) followed in effectiveness with weed density of $61.0 \mathrm{~m}^{-2}$ and weed dry weight of $132.0 \mathrm{~g} \mathrm{~m}^{-2}$ as well as mungbean seed yield of $2282 \mathrm{~kg} \mathrm{ha}^{-1}$. As much as irrigation frequencies are concerned, the least weed density $\left(82.0 \mathrm{~m}^{-2}\right)$ was recorded in two irrigations but optimal seed yield $\left(1965 \mathrm{~kg} \mathrm{ha}^{-1}\right)$ of mungbean was noted in three irrigations. Hence, the results suggested that combination of plants extracts and herbicides at reduced doses controlled weeds efficiently and optimum enhancement in seed yield of mungbean was noticed at three irrigations.
\end{abstract}

Keywords: Allelopathy, bermuda grass, seed yield, sunflower, weeds

Citation: Korejo, M.N.; M.N. Kandhro; A.A. Soomro and N.A. Wahocho. 2021. Weed Management And Yield Improvement In Mungbean Through Allelopathic Action Of Sunflower And Bermuda Grass Extracts In Conjunction With Irrigation Frequencies. Pak. J. Weed Sci. Res., 27 (3):307-320.

\footnotetext{
${ }^{1}$ Department of Agronomy, ${ }^{2}$ Department of Horticulture; Sindh Agriculture University, Tandojam, Pakistan *Corresponding author: naeemkorejo@hotmail.com
} 
308 Muhammad Naeem Korejo, Muhammad Nawaz Kandhro et. al. Weed Management....

\section{Introduction}

Mungbean (Vigna radiata L.) is an essential pulse crop worldwide including Pakistan (Arain, 2012). Mungbean seeds are mostly rich in protein, which contain about 20.97-31.32\% protein (Anwar et al., 2007). Mungbean seed contains protein double than cereals (Chen et al., 2014) and significantly higher protein content than observed for conventional root crops (Kudre et al., 2013). It is cooked, processed, roasted, sprouted or grinded and it is also most essential pulses grown for edible seed. It is a source of high-quality protein for human consumption and can serve as a multipurpose crop, if harvest residues are used as fodder or green manure (Pataczek et al., 2018). It is annually cultivated on an area of 162.4 thousands ha with total production of 122.0 thousands tones in the country (GoP, 2018).

Weeds are considered as the most harmful to agricultural production among biotic constraints. Weeds also indirectly influence the crop production through competing with crop in resources, sheltering crop pests, interfering in water management, reduced yield and quality, and subsequently raising the price of processing (Zimdahl, 2013). Weeds interfere with crop plants not only through competition but also with allelopathy (Javaid et al., 2007; Kandhro et al., 2016). Gharde et al. (2018) reported actual yield losses of $13-43 \%$ in mungbean due to weeds whereas Mirjha et al. (2013) noticed it as ranging from $30-85 \%$. The losses in yield of mungbean and other crops depend upon weed species, their densities and crop weed competition period (Siddiqui et al., 2010). Sharma and Singh (2011) claimed that competition of weeds and crops for water, nutrients and other growth traits, and in lack of an effective weed reducing measures, weeds remove large quantity of applied nutrients resulting in more loss in yield and conditions become severe under its insufficiency. Therefore, it is necessary to discover new weed control methods, which should be natural, environment friendly and effective. Allelopathy is one of the environment friendly and cheapest approaches for weeds supervision (Iqbal and Cheema, 2009; Shafiq et al., 2013). Many recent studies have shown that noxious weeds such as parthenium can be controlled by using extracts of allelopathic weeds namely Ageratum conyzoides and Coronopus didymus (Javaid et al., 2020; Javaid and Khan, 2020). Sunflower (Helianthus annuus L.) has been largely studied due to allelopathic nature (Kamal, 2011) and sustainable weed reduction approach (Javaid et al., 2006; Jabran et al., 2015). Sunflower being an important allelopathic crop releases glycosides, sorgoleone, alkaloids, phenolics, flavonoids and terpeniods allelopathic compounds that actively reduced the growth of near plant species due to highly allelopathic nature (Anjum and Bajwa, 2008), and they affect germination of seeds (Hozayn et al., 2011). Weed density and biomass is reduced significantly by using allopathic crops and weeds (Cheema and Khaliq, 2000; Javaid et al., 2010, 2011). Allelopathy has been effectively utilized to control weeds in various crops including mungbean fields. The yield increased from 15 to $25 \%$ by controlling weeds through allelopathic plant extract (Cheema et al., 2013).

The Bermuda grass plant material contains allelopathic compounds with phytotoxic efficacy. The many water soluble allelopathic compounds such as caffeic acids, phenolic acid, vanillic, ferulic and pcumaric are released by this weed. The crop growth and yield may be affected by availability of this weed in field. Furthermore, allelopathic material of bermuda grass could be used to reduce weeds under field environment (Kandhro et al., 2015). Bermuda grass extract had powerful allelopathic and inhibitory effects on various characters of basil and common purslane. Mahmoodzadeh and Mahmoodzadeh (2014) exposed that all Labiatae species seed germination was completely reduced by applications more than $2 \%$ rhizome bermuda grass extract. The extract had powerful reduction effect on root expanded of seedling in legumes 
and Labiatae to shoot expanded in Poacae. Bermuda grass rhizomes may be a source for bio-herbicide against sorghum halepense which will assist to reduced invasive plants. The effects of this study support the observation of Yarnia et al. (2010) who described that crop growth significantly reduced by bermuda grass material releases allelopathic chemical.

Sufficient water must be used timely to achieve more grain yield under irrigated areas. So, water use of plant and periods is fundamental to conclude that flora is vulnerable for water nearby watering application periods to enhance yield in a sufficient area. It is most essential pulses have noticeable wetness responsive step of development in relation to grain yield. During pod and seed formation, the moisture applied is very essential. Weed density and dry weight were considerably influenced by the quantity of water applications in all the mulching, hand weeding and weedy plots (Towa and Xiangping, 2014). Mahbod et al. (2009) stated that the yield improved significantly by increase in water application levels from three to six and appropriate weed-reduction procedures can improve the effectiveness of utilization of water in crop. Nadeem et al. (2014) reported weed population and dry weight increased with increase in water application levels. Proper water management is the most important factor in controlling weeds during crop production as well as hand weeding.

\section{MATERIALS AND METHODS}

To examine the weed inhibition and yield enhancement in mungbean through allelopathic action of sunflower and bermuda grass extract in conjunction with irrigation frequencies, the study was carried out at Sindh Agriculture University Tandojam, Pakistan in spring seasons of 2019 \& 2020. The design of experiment was split plot design having factorial arrangements with four replications. The area of treatment units was $4 \mathrm{~m} \times 4 \mathrm{~m}$ $\left(16 \mathrm{~m}^{-2}\right)$. The texture of soil appeared silty clay loam, with limited nitrogen $(0.021 \%)$, low organic matter $(0.42 \%)$ and high in
$\mathrm{pH}$ (8.24). The experiment consisted of Two Factors. The main-plot comprised of 3 irrigation regimes $(2,3 \& 4$ irrigations) and Sub-plot consisted of weed control practices (weedy check-untreated, sunflower extract @ $30 \mathrm{~L} \mathrm{ha}^{-1}$, bermuda grass extract @ $30 \mathrm{~L} \mathrm{ha}^{-1}$, sunflower + bermuda grass extract each @ $15 \mathrm{~L} \mathrm{ha}^{-1}$, hand weeding @ 30 days after sowing, atlantis @ $0.2 \mathrm{~L} \mathrm{ha}^{-1}+$ puma super @ $0.312 \mathrm{~L} \mathrm{ha}^{-1}$, sunflower extract: @15 L ha1 + atlantis: $0.1 \mathrm{~L} \mathrm{ha}^{-1}+$ puma super @ $0.156 \mathrm{~L} \mathrm{ha}^{-1}$, bermuda grass extract @ 15 L ha ${ }^{-1}+$ atlantis: $0.1 \mathrm{~L} \mathrm{ha}^{-1}+$ puma super @ $0.156 \mathrm{~L} \mathrm{ha}^{-1}$ ). The extracts and herbicides were applied as per respective treatment after first irrigation. The seeds of mungbean were sown on drilling method in respective plots by single coulter hand drill. Nitrogen and phosphorus were applied at recommended dose of 25-50 kg ha-1 in urea form and DAP. Total DAP and 1/2 Urea were used during land preparation and remaining Urea was used at the time of $2^{\text {nd }}$ irrigation. The crop earthing-up was exercised 2 times after $1^{\text {st }}$ watering and second after $2^{\text {nd }}$ watering application.

\section{Measurement and observations}

Weed and Crop collection data

Weed density $\left(\mathbf{m}^{-2}\right)$ : Weeds were counted at 60 DAS. A wooden frame of one meter square was utilized to note total number of weeds per meter square.

Weed density $\left(\mathrm{m}^{-2}\right)=$ Total No. of weeds $\left(\mathrm{m}^{-2}\right)$ for given treatment /No. of replications

Weed dry weight $\left(\mathbf{g ~ m}^{-2}\right)$ : The weed dry weight was collected from 3 locations of every unit. Weeds were harvested at floor level and dried up through oven for 48 hours at $70{ }^{\circ} \mathrm{C}$ to note dry weed weight.

Seed germination $\left(\mathbf{m}^{-2}\right)$ : Ten days after sowing, seed germination per square meter was collected by counting the seedlings of mungbean.

Leaf area index: The data of leaf area index was noted from selected 5 plants when crop was in peak vegetative growth by the formula: 
310 Muhammad Naeem Korejo, Muhammad Nawaz Kandhro et. al. Weed Management....

Leaf area index $=$ Leaf area per plant $\left(\mathrm{cm}^{2}\right)$

Ground area per plant $\left(\mathrm{cm}^{2}\right)$

Pods plant ${ }^{-1}$ : Pods per plant were counted from selected five plants in every plot and average was recorded at maturity.

Seed weight pod $^{-1}(\mathbf{g})$ : The collected seeds of five randomly chosen pods were weighed individually and the weight of grains per pod average was recorded in grams.

Biological yield ( $\mathbf{k g ~ h a}^{-1}$ ): Data of crop biological yield was noted as per following formula:

Biological yield $=\underline{\text { Crop biological yield per plot }(\mathrm{kg})} \times 1000$

Area per plot $\left(\mathrm{m}^{2}\right)$

Seed yield ( $\mathbf{~ g ~ h a}^{-\mathbf{1}}$ ): Data regarding seed yield of mungbean was noted from harvested plants for crop biological yield by using the formula:

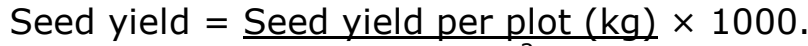
Area per plot $\left(\mathrm{m}^{2}\right)$

\section{Statistical analysis}

The data was statistically analyzed following ANOVA technique using software Statistix version 8.1 (Statistix, 2006). The least significant difference (LSD) test was used at alpha 0.05 for comparing differences of treatments.

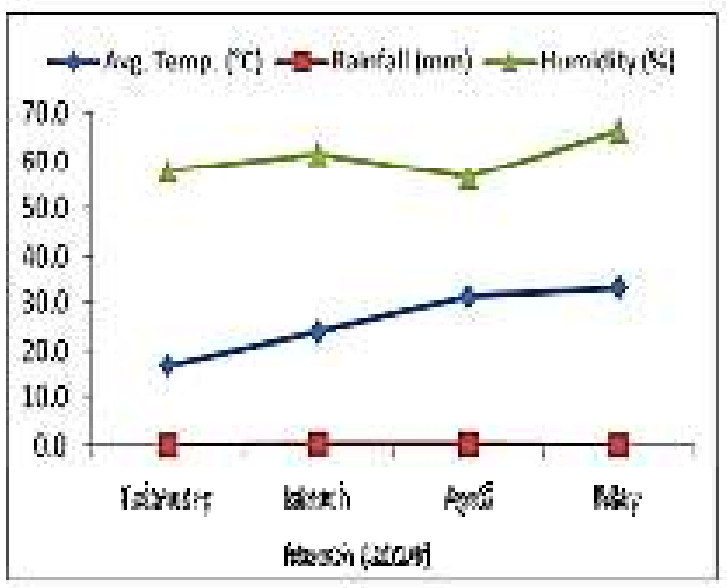

\section{Meteorological data}

During experimental season the meteorological data of Tandojam of 2 years (2019 and 2020) were collected from Meteorological Observatory Station, DRIP, Tandojam. The information of meteorological data on monthly basis for February, March, April and May regarding standard temperature $\left({ }^{\circ} \mathrm{C}\right)$, Rainfall $(\mathrm{mm})$ and humidity (\%) are mentioned in Fig.1

Fig. 1. Meteorological data of Tendojam during crop season (2019 and 2020) 


\section{RESULT AND DISCUSSIONS}

\section{Weed density $\left(\mathrm{m}^{-2}\right)$}

The weed density $\left(\mathrm{m}^{-2}\right)$ of mungbean differed significantly under study (Table 1). Combination of sunflower extract @ 15 $\mathrm{L} \mathrm{ha}^{-1}+$ herbicides (atlantis @ $0.1 \mathrm{~L} \mathrm{ha}^{-1}+$ puma super @ $0.156 \mathrm{~L} \mathrm{ha}^{-1}$ ) resulted in lowest $\left(52 \mathrm{~m}^{-2}\right)$ weed density followed by integration of bermuda grass extract @ 15 $\mathrm{L} \mathrm{ha}{ }^{-1}+$ herbicides (atlantis @ $0.1 \mathrm{~L} \mathrm{ha}^{-1}$ + puma super @ $0.156 \mathrm{~L} \mathrm{ha}^{-1}$ ) with weed density of $61 \mathrm{~m}^{-2}$ whereas highest $\left(141 \mathrm{~m}^{-}\right.$ ${ }^{2}$ ) weed density was noted in weedy check (untreated). Irrigation frequencies also significantly affected the weed density. Two irrigations (30 and 60 DAS) gave lesser weed density $\left(81 \mathrm{~m}^{-2}\right)$ as compared to three and four irrigations ( 85 and 88 weed density $\mathrm{m}^{-2}$ ). Significant effects for interaction of weed control practices $x$ irrigation frequencies were also observed for weed density. Sunflower releases many allelopathic compounds that influence the development of neighboring flora (Anjum and Bajwa, 2008) and they affect germination of seeds (Hozayn et al., 2011). Weed density is reduced ranging from 19 to $49 \%$, by using allopathic plants (Cheema and Khaliq, 2000). Towa and Xiangping (2014) reported that the weed density $t$ were a lot influenced through the quantity of water under mulching, hand weeding and weedy plots.

Table 1. Weed density $\left(\mathrm{m}^{-2}\right)$ and weed dry weight $\left(\mathrm{g} \mathrm{m}^{-2}\right)$ in mungbean as affected by allelopathic plants extract and other practices under different irrigation regimes

\begin{tabular}{|c|c|c|c|c|c|c|c|c|}
\hline \multirow{3}{*}{$\begin{array}{l}\text { Weed control } \\
\text { practices } \\
(\mathrm{WCP})\end{array}$} & \multicolumn{3}{|c|}{ Weed density $\left(\mathrm{m}^{-2}\right)$} & \multirow[t]{3}{*}{ Mean } & \multicolumn{3}{|c|}{ weed dry weight $\left(\mathrm{g} \mathrm{m}^{-2}\right)$} & \multirow[t]{3}{*}{ Mean } \\
\hline & \multicolumn{3}{|c|}{ Irrigation regimes } & & \multicolumn{3}{|c|}{ Irrigation regimes } & \\
\hline & 2 & 3 & 4 & & 2 & 3 & 4 & \\
\hline WC & 137 & 141 & 145 & $141 \mathrm{~A}$ & 378 & 388 & 398 & $388 \mathrm{~A}$ \\
\hline SE: $30 \mathrm{~L} \mathrm{ha}^{-1}$ & 87 & 91 & 95 & $91 \mathrm{C}$ & 250 & 260 & 271 & $260 \mathrm{C}$ \\
\hline BE: $30 \mathrm{~L} \mathrm{ha}^{-1}$ & 99 & 103 & 108 & $103 \mathrm{~B}$ & 281 & 292 & 303 & $292 B$ \\
\hline $\begin{array}{l}\text { SE: +BE: } 15 \mathrm{~L} \mathrm{ha}^{-1} \\
\text { (each) }\end{array}$ & 78 & 81 & 84 & $81 \mathrm{D}$ & 218 & 228 & 239 & $228 \mathrm{D}$ \\
\hline HE: 30 DAS & 75 & 76 & 77 & $76 \mathrm{E}$ & 185 & 196 & 207 & $196 \mathrm{E}$ \\
\hline $\begin{array}{l}\text { A: } 0.2 \mathrm{~L} \mathrm{ha}^{-1}+ \\
\text { P: } 0.312 \mathrm{~L} \mathrm{ha}^{-1}\end{array}$ & 68 & 71 & 74 & $71 \mathrm{~F}$ & 154 & 164 & 175 & $164 \mathrm{~F}$ \\
\hline $\begin{array}{l}\text { SE:15 L ha }{ }^{-1}+A: 0.1 \\
L h a^{-1}+P: 0.156 L_{h a}^{-1}\end{array}$ & 48 & 52 & 55 & $52 \mathrm{H}$ & 89 & 100 & 110 & $100 \mathrm{H}$ \\
\hline $\mathrm{BE}: 15 \mathrm{~L} \mathrm{ha}^{-1}+\mathrm{A}: 0.1$ & 58 & 61 & 64 & $61 \mathrm{G}$ & 121 & 132 & 143 & $132 \mathrm{G}$ \\
\hline
\end{tabular}


312 Muhammad Naeem Korejo, Muhammad Nawaz Kandhro et. al. Weed Management....

\begin{tabular}{|c|c|c|c|c|c|c|c|c|c|}
\hline $\mathrm{L} \mathrm{ha}{ }^{-1}+\mathrm{P}: 0.1$ & & & & & & & & & \\
\hline Means & $81 \mathrm{C}$ & $85 B$ & $8 \varepsilon$ & & -- & $210 \mathrm{C}$ & $220 \mathrm{~B}$ & $231 \mathrm{~A}$ & -- \\
\hline Variable & $S E$ & P-val & & LSL & D. $(5 \%)$ & $S E$ & P-values & & D. $(5 \%)$ \\
\hline Irrigation (I) & $\begin{array}{c}0.7526 \\
0\end{array}$ & 0.003 & & & .8414 & 0.06080 & $0.0000 * *$ & & 0.1487 \\
\hline WCP & 0.8267 & 0.000 & & & .6520 & 1.1666 & $0.0000 * *$ & & 2.3312 \\
\hline$I \times$ WCP & 1.4319 & 0.000 & & & .8614 & 2.0205 & $0.0000 * *$ & & 4.0377 \\
\hline
\end{tabular}

$W C=$ Weedy check, $\mathrm{SE}=$ Sunflower extract, $\mathrm{BE}=$ Bermuda grass extract, $\mathrm{HW}=\mathrm{Hand}$ weeding, $\mathrm{A}=$ Atlantis, $\mathrm{P}=$ Puma super $\mathrm{NS}=$ Non-significant at $\mathrm{P}_{0.05} ; *$ Significant at $\mathrm{P}_{0.05} ; * *=$ Significant at $\mathrm{P}_{0.01}$.

\section{Weed dry weight $\left(\mathrm{g} \mathrm{m}^{-2}\right)$}

All treatments significantly reduced weed dry weight compared with weedy check (untreated) (Table 1 ). The data revealed that the mixture of sunflower extract @ $15 \mathrm{~L} \mathrm{ha}^{-1}+$ herbicides (atlantis @ $0.1 \mathrm{~L} \mathrm{ha}^{-}$ ${ }^{1}+$ puma super @ $0.156 \mathrm{~L} \mathrm{ha}^{-1}$ ) provided reduced $\left(100 \mathrm{~g} \mathrm{~m}^{-2}\right)$ weed dry weight followed by incorporation of bermuda grass extract @ $15 \mathrm{~L} \mathrm{ha}^{-1}$ with herbicides (atlantis @ $0.1 \mathrm{~L} \mathrm{ha}^{-1}+$ puma super @ $0.156 \mathrm{~L} \mathrm{ha}^{-1}$ ) resulting in $132 \mathrm{~g} \mathrm{~m}^{-2}$ weed dry weight against weed dry of $388 \mathrm{~g} \mathrm{~m}^{-2}$ recorded in weedy check (untreated). Irrigation levels also significantly affected the weed dry weight. Two irrigations (30 and 60 DAS) distinguished the minimum weed dry weight $\left(210 \mathrm{~g} \mathrm{~m}^{-2}\right)$ followed by three irrigations $\left(220 \mathrm{~g} \mathrm{~m}^{-2}\right)$ while maximum weed dry weight $\left(231 \mathrm{~g} \mathrm{~m}^{-2}\right.$ ) was noted in four irrigations. Substantial interactive effect of weed control practices $x$ irrigation levels was documented for weed dry weight. Towa and Xiangping (2014) described that the weed dry weight were significantly affected by the quantity of water under all the mulching, hand weeding and weedy plots.

\section{Seed germination $\left(\mathrm{m}^{-2}\right)$}

Various weed control practices reflected a positive influence in seed germination of mungbean. The weed control practices showed significant $(P<0.05)$ results for seed germination (Table 2). The maximum (36.09 $\mathrm{m}^{-2}$ ) seed germination was noted under the combination of sunflower extract @ $15 \mathrm{~L} \mathrm{ha}^{-1}$ with herbicides (atlantis @ $0.1 \mathrm{~L} \mathrm{ha}^{-1}+$ puma super @ $0.156 \quad \mathrm{~L}^{-1}$ ) trailed by integration of bermuda grass extract @ 15 L ha-1 with herbicides (atlantis @ $0.1 \mathrm{~L} \mathrm{ha}^{-}$ $1+$ puma super @ $0.156 \mathrm{~L} \mathrm{ha}^{-1}$ ) with $35.01 \mathrm{~m}^{-2}$ seed germination. However, minimum $\left(26.36 \mathrm{~m}^{-2}\right.$ ) seed germination was found under weedy check (untreated). Among irrigation frequencies, four irrigations noticed in maximum (32.40 $\left.\mathrm{m}^{-2}\right)$ seed germination as compared to three irrigations and two irrigations (32.04 and $31.68 \mathrm{~m}^{-2}$ ). Remarkable effect of interaction of weed control practices $\times$ irrigation frequencies was observed for seed germination $\left(\mathrm{m}^{-2}\right)$. The water extracts of allelopathic crops caused substantial reduction in germination and growth of tested summer 
weeds such as jungle rice (Echinochloa colonum L.), bermuda grass (Cynodon dactylon L.) and purple nutsedge (Cyperus rotundus L.) (Kandhro et al., 2015). These results support the findings of Maharjan et al. (2007) who found that leaf water extracts of Parthenium hysterophorus exhibited significant reduction effects on germination of seeds and seedling development, Similarly Bajwa et al. (2004) reported that aqueous extract from shoot of Parthenium reduced the germination and seedling growth.

Table 2. Seed germination $\left(\mathrm{m}^{-2}\right)$ and leaf area index in mungbean as affected by allelopathic plants extract and other practices under different irrigation regimes

\begin{tabular}{|c|c|c|c|c|c|c|c|c|}
\hline \multirow[t]{2}{*}{$\begin{array}{l}\text { Weed control } \\
\text { practices } \\
(\mathrm{WCP})\end{array}$} & \multicolumn{3}{|c|}{$\begin{array}{c}\text { Seed germination }\left(\mathrm{m}^{-}\right. \\
\left.{ }^{-}\right)\end{array}$} & Mean & \multicolumn{3}{|c|}{ leaf area index } & \multirow[t]{2}{*}{ Mean } \\
\hline & 2 & 3 & 4 & & 2 & 3 & 4 & \\
\hline WC & 26.00 & 26.36 & 26.73 & $26.36 \mathrm{H}$ & 3.10 & 3.14 & 3.18 & $3.14 \mathrm{H}$ \\
\hline SE: $30 \mathrm{~L} \mathrm{ha}^{-1}$ & 30.33 & 30.69 & 31.05 & $30.69 \mathrm{~F}$ & 3.58 & 3.63 & 3.68 & $3.63 \mathrm{~F}$ \\
\hline BE: $30 \mathrm{~L} \mathrm{ha}^{-1}$ & 29.25 & 29.61 & 29.97 & $29.61 \mathrm{G}$ & 3.46 & 3.50 & 3.54 & $3.50 \mathrm{G}$ \\
\hline $\begin{array}{l}\text { SE: +BE: } 15 \mathrm{~L} \mathrm{ha}^{-1} \\
\text { (each) }\end{array}$ & 31.41 & 31.77 & 32.13 & $31.77 \mathrm{E}$ & 3.73 & 3.78 & 3.83 & $3.78 \mathrm{E}$ \\
\hline HE: 30 DAS & 32.49 & 32.85 & 33.21 & $32.85 \mathrm{D}$ & 3.88 & 3.93 & 3.98 & $3.93 \mathrm{D}$ \\
\hline $\begin{array}{l}\text { A: } 0.2 \mathrm{~L} \mathrm{ha}^{-1}+ \\
\text { P: } 0.312 \mathrm{~L} \mathrm{ha}^{-1}\end{array}$ & 33.57 & 33.93 & 34.29 & $33.93 \mathrm{C}$ & 4.03 & 4.08 & 4.13 & $4.08 \mathrm{C}$ \\
\hline $\begin{array}{l}\text { SE: } 15 \text { L ha }{ }^{-1}+A: 0.1 \\
L h a^{-1}+P: 0.156 L \\
h a^{-1}\end{array}$ & 35.73 & 36.10 & 36.46 & $36.09 \mathrm{~A}$ & 4.33 & 4.38 & 4.88 & $4.53 \mathrm{~A}$ \\
\hline $\begin{array}{l}\text { BE: } 15 \mathrm{~L} \mathrm{ha}^{-1}+\mathrm{A}: 0.1 \\
\mathrm{~L} h \mathrm{~h}^{-1}+\mathrm{P}: 0.156 \mathrm{~L} \\
\mathrm{ha^{-1 }}\end{array}$ & 34.65 & 35.01 & 35.37 & $35.01 \mathrm{~B}$ & 4.18 & 4.23 & 4.28 & $4.23 \mathrm{~B}$ \\
\hline Means & $\begin{array}{c}31.68 \\
C\end{array}$ & $\begin{array}{c}32.04 \\
\text { B }\end{array}$ & $\begin{array}{c}32.40 \\
\text { A }\end{array}$ & -- & $3.79 \mathrm{C}$ & $3.83 \mathrm{~B}$ & 3.94 & -- \\
\hline Variable & S E & P-V & alues & $\begin{array}{l}\text { LSD. } \\
(5 \%)\end{array}$ & S E & $P-v$ & lues & LSD. (5\%) \\
\hline
\end{tabular}


314 Muhammad Naeem Korejo, Muhammad Nawaz Kandhro et. al. Weed Management....

\begin{tabular}{|l|c|c|c|c|c|c|}
\hline Irrigation (I) & 2.94604 & $0.0000 * *$ & 0.0072 & 6.37403 & $0.0000 * *$ & 0.0156 \\
\hline WCP & 1.31103 & $0.0000 * *$ & 1.0030 & 0.0365 & $0.0000 * *$ & 0.0729 \\
\hline I x WCP & 2.27103 & $0.0428 *$ & 4.53903 & 0.0632 & $0.0000 * *$ & 0.1262 \\
\hline
\end{tabular}

$\mathrm{WC}=$ Weedy check, $\mathrm{SE}=$ Sunflower extract, $\mathrm{BE}=$ Bermuda grass extract, $\mathrm{HW}=$ Hand weeding, $\mathrm{A}=$ Atlantis, $\mathrm{P}=$ Puma super $\mathrm{NS}=$ Non-significant at $\mathrm{P}_{0.05} ;{ }^{*}=$ Significant at $\mathrm{P}_{0.05} ; * *=$

Significant at $\mathrm{P}_{0.01}$.

\section{Leaf area index}

The data (Table 2) revealed that various weed control practices significantly improved leaf area index of mungbean in contrast to control (weedy check). The data of leaf area index was noted high (4.53) in plot under combination of sunflower extract @ $15 \mathrm{~L} \mathrm{ha}^{-1}$ with herbicides (atlantis @ $0.1 \mathrm{~L} \mathrm{ha}^{-1}+$ puma super @ $0.156 \mathrm{~L} \mathrm{ha}^{-1}$ ) followed by integration of bermuda grass extract @ 15 L ha-1 with herbicides (atlantis @ $0.1 \mathrm{~L} \mathrm{ha}^{-}$ 1 + puma super @ $\left.0.156 \mathrm{~L} \mathrm{ha}^{-1}\right)(4.23)$ and low (3.14) was noticed in weedy check (untreated). Four irrigations produced maximum (3.94) leaf area index trailed by three irrigations with 3.83 leaf area index. Nevertheless, less (3.79) leaf area index was noticed in two irrigations. Significant interaction of weed control practices $x$ irrigation frequencies were observed for leaf area index.

\section{Pods plant ${ }^{-1}$}

The data (Table 3 ) regarding pods per plant showed significant effect of weed control practices. Combination of sunflower extract @ $15 \mathrm{~L} \mathrm{ha}^{-1}+$ herbicides (atlantis @ $0.1 \mathrm{~L} \mathrm{ha}^{-1}+$ puma super @ $0.156 \mathrm{~L} \mathrm{ha}^{-1}$ ) gave significantly highest pods plant $^{-1}$ of 36.26 followed by integration of bermuda grass extract @ 15 L ha ${ }^{-1}+$ herbicides (atlantis @ $0.1 \mathrm{~L} \mathrm{ha}^{-1}$ + puma super @ $0.156 \mathrm{~L} \mathrm{ha}^{-1}$ ) which produced (34.76) whereas lowest (23.07) pods plant ${ }^{-1}$ were obtained in weedy check (untreated). It is also noticeable that irrigation levels influenced significantly on pods plant $^{-1}$. Four irrigations produced maximum (31.24) pods plant ${ }^{-1}$ followed by three irrigations with 30.72 pods plant $^{-1}$. Nonetheless, least (30.25) pod plant $^{-1}$ were noted in two irrigations. The interaction of weed control practices $x$ irrigation frequencies was found significant for pods plant ${ }^{-1}$. Din and Parvin (2013) reported that water stress affects different growth and developmental stages of mungbean. The increase in watering application caused significant increase in the number of pods plant ${ }^{-1}$ (Albinet, 2000). 
Pak. J. Weed Sci. Res., 27(3): 307-320, 2021315

Table 3. Pods plant ${ }^{-1}$ and seed weight $\operatorname{pod}^{-1}(\mathrm{~g})$ in mungbean as affected by allelopathic plants extract and other practices under different irrigation regimes

\begin{tabular}{|c|c|c|c|c|c|c|c|c|c|c|}
\hline \multirow{3}{*}{$\begin{array}{l}\text { Weed control } \\
\text { practices } \\
(\mathrm{WCP})\end{array}$} & \multicolumn{4}{|c|}{ Pods plant $^{-1}$} & \multirow[t]{3}{*}{ Mean } & \multicolumn{4}{|c|}{ seed weight pod $^{-1}(\mathrm{~g})$} & \multirow[t]{3}{*}{ Mean } \\
\hline & \multicolumn{4}{|c|}{ Irrigation regimes } & & \multicolumn{4}{|c|}{ Irrigation regimes } & \\
\hline & 2 & & 3 & 4 & & 2 & & 3 & 4 & \\
\hline WC & 22.53 & & .01 & 23.67 & $23.07 \mathrm{H}$ & 0.42 & & 42 & 0.43 & $0.42 \mathrm{H}$ \\
\hline SE: $30 \mathrm{~L} \mathrm{ha}^{-1}$ & 28.41 & & .89 & 29.37 & $28.89 \mathrm{~F}$ & 0.51 & & 52 & 0.53 & $0.52 \mathrm{~F}$ \\
\hline BE: $30 \mathrm{~L} \mathrm{ha}^{-1}$ & 27.03 & & .45 & 27.93 & $27.47 \mathrm{G}$ & 0.48 & & 49 & 0.50 & $0.49 \mathrm{G}$ \\
\hline $\begin{array}{l}\text { SE: +BE: } 15 \mathrm{~L} \mathrm{ha}^{-1} \\
\text { (each) }\end{array}$ & 29.86 & & .35 & 30.84 & $30.35 \mathrm{E}$ & 0.54 & & 55 & 0.56 & $0.55 \mathrm{E}$ \\
\hline HE: 30 DAS & 31.33 & & .82 & 32.31 & $31.82 \mathrm{D}$ & 0.57 & & 58 & 0.59 & $0.58 \mathrm{D}$ \\
\hline $\begin{array}{l}\text { A: } 0.2 \mathrm{~L} \mathrm{ha}^{-1}+ \\
\text { P: } 0.312 \mathrm{~L} \mathrm{ha}^{-1}\end{array}$ & 32.80 & & .29 & 33.78 & $33.29 \mathrm{C}$ & 0.60 & & 61 & 0.62 & $0.61 \mathrm{C}$ \\
\hline $\begin{array}{l}\text { SE:15 L ha }{ }^{-1}+A: 0.1 \\
L h^{-1}+P: 0.156 \text { L } \\
h a^{-1}\end{array}$ & 35.74 & & .23 & 36.82 & $36.26 \mathrm{~A}$ & 0.66 & & 67 & 0.68 & $0.67 \mathrm{~A}$ \\
\hline $\begin{array}{l}\text { BE: } 15 \text { L ha }{ }^{-1}+A: 0.1 \\
L h a^{-1}+P: 0.156 L \\
h a^{-1}\end{array}$ & 34.27 & & .76 & 35.25 & $34.76 \mathrm{~B}$ & 0.63 & & 64 & 0.65 & $0.64 \mathrm{~B}$ \\
\hline Means & $\begin{array}{c}30.25 \\
\mathrm{C}\end{array}$ & & $\begin{array}{l}.72 \\
B\end{array}$ & $\begin{array}{c}31.24 \\
\text { A }\end{array}$ & -- & $0.55 \mathrm{C}$ & 0.5 & $6 \mathrm{~B}$ & $0.57 \mathrm{~A}$ & -- \\
\hline Variable & S E & & $P-v$ & lues & $\begin{array}{l}\text { LSD. } \\
(5 \%)\end{array}$ & S E & & $P-1$ & alues & LSD. (5\%) \\
\hline Irrigation (I) & $0.017 \varepsilon$ & & 0.00 & $00 * *$ & 0.0436 & 2.8910 & & 0.0 & $001 * *$ & 0.0070 \\
\hline WCP & 0.042 & & 0.00 & $00 * *$ & 0.0848 & 8.214 & & 0.0 & $000 * *$ & 0.0164 \\
\hline$I \times W C P$ & 0.073 & & 0.00 & $33 * *$ & 0.1468 & 0.014 & & 0.0 & $000 * *$ & 0.0284 \\
\hline
\end{tabular}

$\mathrm{WC}=$ Weedy check, $\mathrm{SE}=$ Sunflower extract, $\mathrm{BE}=$ Bermuda grass extract, $\mathrm{HW}=$ Hand weeding, $\mathrm{A}=$ Atlantis, $\mathrm{P}=$ Puma super $\mathrm{NS}=$ Non-significant at $\mathrm{P}_{0.05} ; *$ Significant at $\mathrm{P}_{0.05} ; * *=$ Significant at $P_{0.01}$. 


\section{Seed weight $\operatorname{pod}^{-1}(g)$}

The combination of sunflower extract @ $15 \mathrm{~L} \mathrm{ha}^{-1}$ with herbicides (atlantis @ $0.1 \mathrm{~L}$ $\mathrm{ha}^{-1}+$ puma super @ $0.156 \mathrm{~L} \mathrm{ha}^{-1}$ ) revealed significantly greatest seed weight pod $^{-1}$ of $0.67 \mathrm{~g}$. The integration of bermuda grass extract @ $15 \mathrm{~L} \mathrm{ha}^{-1}$ with herbicides (atlantis @ $0.1 \mathrm{~L} \mathrm{ha}^{-1}+$ puma super @ $0.156 \mathrm{~L} \mathrm{ha}^{-1}$ ) followed and created seed weight pod $^{-1}$ of $0.64 \mathrm{~g}$ and lowest $(0.42 \mathrm{~g})$ seed weight pod $^{-1}$ was achieved in weedy check (untreated). Seed weight pod $^{-1}$ was significant regarding irrigation frequencies effects. Four irrigations resulted in more $0.57 \mathrm{~g}$ seed weight pod $^{-1}$ followed by three irrigations which produced seed weight of $0.56 \mathrm{~g} \mathrm{pod}^{-1}$. However, less $0.55 \mathrm{~g}$ seed weight pod $^{-1}$ was recorded in two irrigations. The interaction of weed control practices $x$ irrigation frequencies was found substantial for seed weight pod $^{-1}$ (Table 3).

\section{Biological yield $\left(\mathrm{kg} \mathrm{ha}^{-1}\right)$}

The weed control practices noticed significant $(P<0.05)$ results for crop biological yield (Table 4) of mungbean. The more (4945 $\mathrm{kg} \mathrm{ha}^{-1}$ ) biological yield was found under the combination of sunflower extract @ $15 \mathrm{~L} \mathrm{ha}^{-1}$ with herbicides (atlantis @ $0.1 \mathrm{~L} \mathrm{ha}^{-1}+$ puma super @ $0.156 \mathrm{~L} \mathrm{ha}^{-1}$ ) followed by integration of bermuda grass extract @ 15 L ha ${ }^{-1}$ with herbicides (atlantis @ $0.1 \mathrm{~L} \mathrm{ha}^{-}$ 1 + puma super @ $0.156 \mathrm{~L} \mathrm{ha}^{-1}$ ) resulting in $4815 \mathrm{~kg} \mathrm{ha}^{-1}$ and lowest (3874 $\mathrm{kg} \mathrm{ha}^{-1}$ ) biological yield was achieved in weedy check (untreated). Four irrigations provided highest (4564 $\mathrm{kg} \mathrm{ha}^{-1}$ ) biological yield as compared to three and two irrigations (4539 and $4384 \mathrm{~kg} \mathrm{ha}^{-1}$ ). The interactive effects of weed control practices $x$ irrigation frequencies were found significant for biological yield ( $\mathrm{kg}$ $\left.\mathrm{ha}^{-1}\right)$. These findings are similar as shown by Cheema and Khaliq (2000) who exposed increased biological yield due to weed control in wheat. Similarly, Sangakara (1994) reported that the growth and yield of mungbean crop significantly improved by the availability of sufficient soil moisture.

Table 4. Biological yield $\left(\mathrm{kg} \mathrm{ha}^{-1}\right)$ and seed yield $\left(\mathrm{kg} \mathrm{ha}^{-1}\right)$ in mungbean as affected by allelopathic plants extract and other practices under different irrigation regimes

\begin{tabular}{|c|c|c|c|c|c|c|c|c|}
\hline \multirow{3}{*}{$\begin{array}{l}\text { Weed control } \\
\text { practices } \\
(\mathrm{WCP})\end{array}$} & \multicolumn{3}{|c|}{ Biological yield (kg ha } & \multirow[t]{3}{*}{ Mean } & \multicolumn{3}{|c|}{ seed yield $\left(\mathrm{kg} \mathrm{ha}^{-1}\right)$} & \multirow[t]{3}{*}{ Mean } \\
\hline & \multicolumn{3}{|c|}{ Irrigation regimes } & & \multicolumn{3}{|c|}{ Irrigation regimes } & \\
\hline & 2 & 3 & 4 & & 2 & 3 & 4 & \\
\hline WC & 3764 & 3917 & 3942 & $3874 \mathrm{H}$ & 1254 & 1352 & 1383 & $1330 \mathrm{H}$ \\
\hline SE: $30 \mathrm{~L} \mathrm{ha}^{-1}$ & 4236 & 4389 & 4413 & $4346 \mathrm{~F}$ & 1716 & 1813 & 1844 & $1791 \mathrm{~F}$ \\
\hline BE: $30 \mathrm{~L} \mathrm{ha}^{-1}$ & 4119 & 4272 & 4296 & $4229 \mathrm{G}$ & 1596 & 1694 & 1725 & $1671 \mathrm{G}$ \\
\hline SE:+BE: $15 \mathrm{~L} \mathrm{ha}^{-1}$ & 4354 & 4508 & 4533 & $4465 \mathrm{E}$ & 1835 & 1932 & 1963 & $1910 \mathrm{E}$ \\
\hline
\end{tabular}


Pak. J. Weed Sci. Res., 27(3): 307-320, 2021317

\begin{tabular}{|c|c|c|c|c|c|c|c|c|c|c|}
\hline (each) & & & & & & & & & & \\
\hline HE: 30 DAS & 4474 & & 28 & 4653 & $4585 \mathrm{D}$ & 1954 & & 52 & 2083 & 2029 D \\
\hline $\begin{array}{l}\text { A: } 0.2 \mathrm{~L} \mathrm{ha}^{-1}+ \\
\text { P: } 0.312 \mathrm{~L} \mathrm{ha}^{-1}\end{array}$ & 4594 & & 748 & 4763 & $4702 \mathrm{C}$ & 2074 & & 71 & 2203 & $2149 \mathrm{C}$ \\
\hline $\begin{array}{l}\text { SE:15 L ha }{ }^{-1}+A: 0.1 \\
L h^{-1}+P: 0.156 L \\
h a^{-1}\end{array}$ & 4824 & & 88 & 5024 & $4945 \mathrm{~A}$ & 2313 & & 11 & 2449 & $2391 \mathrm{~A}$ \\
\hline $\begin{array}{l}\text { BE: } 15 L^{h} a^{-1}+A: 0.1 \\
L h a^{-1}+P: 0.156 L \\
h a^{-1}\end{array}$ & 4704 & & 358 & 4883 & $4815 B$ & 2193 & & 91 & 2322 & $2269 B$ \\
\hline Means & $\begin{array}{c}4384 \\
\text { B }\end{array}$ & & $39 \mathrm{~A}$ & $4564 \mathrm{~A}$ & -- & $1867 \mathrm{~B}$ & & $5 \mathrm{~A}$ & 1996 & -- \\
\hline Variable & SE & & P-v & alues & $\begin{array}{l}\text { LSD. } \\
(5 \%)\end{array}$ & SE & & $P-V$ & alues & LSD. ( $5 \%)$ \\
\hline Irrigation (I) & 0.7684 & & 0.00 & $00 * *$ & 26.8802 & 0.0920 & & 0.0 & $100 * *$ & 32.2252 \\
\hline WCP & 2.381 & & 0.00 & $00 * *$ & 4.7593 & 0.561 & & 0.0 & $00 * *$ & 1.1226 \\
\hline$I \times W C P$ & 4.125 & & 0.0 & $118^{*}$ & 8.2434 & 0.973 & & 0.0 & $00 * *$ & 1.9444 \\
\hline
\end{tabular}

$\mathrm{WC}=$ Weedy check, $\mathrm{SE}=$ Sunflower extract, $\mathrm{BE}=$ Bermuda grass extract, $\mathrm{HW}=$ Hand weeding, $\mathrm{A}=$ Atlantis, $\mathrm{P}=$ Puma super $\mathrm{NS}=$ Non-significant at $\mathrm{P}_{0.05} ;{ }^{*}=$ Significant at $\mathrm{P}_{0.05} ; * *=$

Significant at $P_{0.01}$.

\section{Seed yield $\left(\mathrm{kg} \mathrm{ha}^{-1}\right)$}

The data (Table 4) exposed that different weed control practices treatments significantly improved seed yield attributes of mungbean in contrast to weedy check (untreated). The combination of sunflower extract @ $15 \mathrm{~L}$ ha-1 with herbicides (atlantis @ $0.1 \mathrm{~L} \mathrm{ha}^{-1}$ + puma super @ $0.156 \quad \mathrm{~L} \quad \mathrm{ha}^{-1}$ ) considerably produced more seed yield (2391 kg ha-1) over integration of bermuda grass extract @ $15 \mathrm{~L} \mathrm{ha}^{-1}+$ herbicides (atlantis @ $0.1 \mathrm{~L} \mathrm{ha}^{-1}+$ puma super @ $0.156 \mathrm{~L} \mathrm{ha}^{-1}$ ) resulting in $2269 \mathrm{~kg}$ $\mathrm{ha}^{-1}$. Nevertheless, lowest $\left(1330 \mathrm{~kg} \mathrm{ha}^{-1}\right.$ ) seed yield was noticed in weedy check (untreated). Seed yield was also significantly inflated by irrigation frequencies. Four irrigations produced greater (1996 kg ha-1) seed yield followed by three irrigations with $1965 \mathrm{~kg} \mathrm{ha}^{-1}$ and lesser $1867 \mathrm{~kg} \mathrm{ha}^{-1}$ seed yield was noted under two irrigations. Significant interactive effects of weed control practices $x$ irrigation frequencies were found for seed yield $\left(\mathrm{kg} \mathrm{ha}^{-1}\right)$. Combined utilization of allelopathic plants extracts is more helpful for controlling weeds as compared to alone (Cheema et al., 2003). Control of weeds through allelopathic plant extract enhanced yield from 15 to 25\% (Cheema et al., 2013). Raza et al. 
(2012) concluded that more seed yield of mungbean crop obtained with optimum watering.

\section{Conclusion}

The present research suggested that combination of sunflower extract with low dose of herbicide (sunflower extract: $15 \mathrm{~L}$ ha ${ }^{-1}+$ atlantis: $0.1 \mathrm{~L} \mathrm{ha}^{-1}+$ puma super: $0.156 \mathrm{~L} \mathrm{ha}^{-1}$ ) reduce weeds and enhanced mungbean seed yield remarkably. Amongst irrigation frequencies, three times application of irrigation was found appropriate for controlling weeds and obtaining optimal seed yield of mungbean along with application of sunflower water extract and reduced dose of herbicides.

\section{Acknowledgments}

This research paper is prepared from $\mathrm{PhD}$ thesis of main author submitted at Sindh Agriculture University Tandojam.

\section{Novelty Statement}

\section{REFERENCES CITED}

Albinet, S. 2000. Effect of irrigation and fertilizer on the growth and yield of mungbean. Field Crop Res., 12:34-40.

Anjum, T. and R. Bajwa. 2008. The effect of sunflower leaf extracts on Chenopodium album in wheat fields in Pakistan. Crop Prot., 26(9): 13901394.

Anwar, F., S. Latif, R. Przybylski, B. Sultana and M. Ashraf. 2007. Chemical composition and antioxidant activity of seeds of different cultivars of mungbean. J. Food Sci., 72(7): S503-510.

Arain, G.N. 2012. Mungbean cultivation in Pakistan. Valley mungbean cultivation in Pakistan. Center pivot irrigation system valley irrigation, Pakitsan Private, Limited.
The present research work investigated the allelopathic effect of sunflower and bermuda grass extract in conjunction with irrigation frequencies on weed management and yield improvement in mungbean for the first time under Tandojam conditions. This could help in weed reduction and yield enhancement in the local conditions.

\section{Author's Contribution}

M.N. Korejo planned experiment, data collection and prepared manuscript. M.N. Kandhro guided scholar as a whole from designing experiment to writing of manuscript. A.A. Soomro provided research facility and helped in manuscript write-up. N.A. Wahocho helped in research material, data analysis and interpretation of results.

\section{Conflict of interest}

There is no conflict of interest among authors.

Bajwa, R., S. Shafique, S. Shafique and A. Javaid. 2004. Effect of foliar spray of aqueous extract of Parthenium hysterophorus on growth of sunflower. Int. J. Agric. Biol., 6(3): 474-478.

Cheema, Z.A., A. Khaliq and R. Hussain. 2003. Reducing herbicide rate in combination with allelopathic sorgaab for weed control in cotton. Int. J. Agric. Biol., 5: 4-6.

Cheema, Z.A., A. Rakha and A. Khaliq. 2000. Use of sorgaab and sorghum mulch for weed management in mungbean. Pak. J. Agric. Sci., 101(37): 3-4.

Cheema, Z.A., M. Farooq and A. Khaliq. 2013. Application of allelopathy in 
crop production: Success story from Pakistan. 6: 113-143.

Chen, M.X., S.X. Zheng, Y.N. Yang, C. Xu, J.S. Liu and W.D. Yang. 2014. Strong seed-specific protein expression from the Vigna radiata storage protein $8 \mathrm{SG}$ alpha promoter in transgenic Arabidopsis seeds. J Biotechnol., 174: 49-56.

Chon, S.U.K. and C. J. Nelson. 2013. Allelopathic dynamics in resource plants. 81-110.

Din, S., and S. Parvin. 2013. Yield performance of mungbean (Vigna radiata L.) as influenced by irrigation. Int. J. Agron. Plant. Prod., 4 (S): 3659-3667.

Gharde,Y., P.K. Singha, R.P. Dubeya and P.K. Guptab. 2018. Assessment of yield and economic losses in agriculture due to weeds in India. Crop Prot., 107: 12-18.

GoP, 2018. Economic survey of Pakistan. 2018. Government of Pakistan Ministry of food, agriculture and livestock division (Economic affairs wing) Islamabad.

Hozayn, M., A.A.A. Monem and E.M.A. Lateef. 2011. Crop residues, an effective tool for improving growth of wheat and suppression of some associated weeds. Allelopathy J., 27(2): 237-344.

Iqbal, J. and Z.A. Cheema. 2009. Response of purple nutsedge (Cyperus rotundus L.) to crop extracts prepared in various solvents. Allelopathy J., 23 (2): 450-452.

Jabran, K., G. Mahajan, V. Sardana and B.S. Chaulan. 2015. Allelopathy for weed control in agricultural systems. Crop Prot., 72(2015): 57-65.

Jamil, M., Z. Ata and A. Khaliq. 2005. Increasing the efficacy of sorghum water extract (sorgaab) by mixing with lower doses of isoproturon to control weeds in wheat. Int. J. Agric. Biol., 7(8): 712-718.

Javaid, A,S. Shafique, R. Bajwa and S. Shafique. 2006. Effect of aqueous extracts of allelopathic crops on germination and growth of
Parthenium hysterophorus (L.). South Afr. J. Bot., 72(4): 609-612.

Javaid, A.,R. Bajwa, N. Rabbani and T. Anjum. 2007. Comparative tolerance of rice (Oryza sativa L.) genotypes to purple nutsedge (Cyperus rotundus L.) allelopathy. Allelopathy J., 20(1): 157-166.

Javaid, A.,S. Shafique, S. Shafique. 2010. Herbicidal effects of extracts and residue incorporation of Datura metel against parthenium weed. Nat. Prod. Res., 24(15): 1426-1437.

Javaid, A.,S. Shafique, S. Shafique. 2011. Management of Parthenium hysterophorus (Asteraceae) by Withania somnifera (Solanaceae). Nat. Prod. Res., 25 (4): 407-416.

Javaid, A. and I.H. Khan. 2020. Potential use of Coronopus didymus in parthenium management. Pak. J. Weed Sci. Res., 26(1): 37-45.

Javaid, N., M.H. Shah, I.H. Khan, A. Javaid and S.M. Waleed. 2020. Herbicidal activity of Ageratum conyzoides against parthenium. Pak. J. Weed Sci. Res., (2):137-146.

Kamal, J. 2011. Impact of allelopathy of sunflower roots extract on physiology of wheat (Triticum aestivum L.). Afr. J. Biotechnol., 10(65): 14465-14477.

Kandhro, M.N., M.A. Ansari, A. Naqi, M. Ibrahim and H.R. Memon. 2015. Laboratory studies on the allelopathic potential of sorghum and sunflower water extract and powder against narrow-leaf summer weeds. Gomal Uni. J. Res., 31: ISSN: 1019-8180.

Kandhro, M.N., A.K. Jalbani, N.A. Wahocho, G.M. Sahito, M. Solangi and Q. Jogi. 2016. Laboratory studies on germinability and seedling growth of cotton crop under the allelopathic influence of purple nutsedge. Pak. J. Weed Sci. Res., 22(3): 407-416.

Kudre, T.G., S. Benjakul and H. Kishimura. 2013. Comparative study on chemical compositions and properties of protein isolates from mung bean, black bean and bambara groundnut. J. Sci. Food Agric., 93(10): 2429-36. 
Maharjan, S., B.B. Shrestha and P.K. Jha. 2007. Allelopathic effects of aqueous extract of leaves of Parthenium hysterophorus (L.) on seed germination and seedling growth of some cultivated and wild herbaceous species. Sci. World, 5(5): 33-39.

Mahbod, M., A.R. Sepaskhah and M. Monfared. 2009. Development of an irrigation scheduling model for winter wheat based on soil water and precipitation. J. Sci. Technol. Agric. Nat. Resour., 13(4-B): 1-12.

Mahmoodzadeh, $\mathrm{H}$. and $M$. Mahmoodzadeh. 2014. Allelopathic effects of Cynodon dactylon (L.) on germination and growth of Triticum aestivum (L.). Ann. Biol. Res., 5(1): 118-123.

Marwat, K.B., Z. Hussain, M. Saeed, B. Gul and S. Noor. 2005. Chemical weed management in wheat at higher altitude. Pak. J. Weed Sci. Res., 11: 103-108.

Mirjha, P.R., S.K. Prasad, M.K. Singh, R.H. Paikra, S. Patel and M. Majumdar. 2013. Effect of weed control measures on weeds, nodulation, growth andyield of greengram (Vigna radiata). Indian J. Agron., 58: 615617.

Nadeem, M.A, A. Tanveer, A. Ali, M. Ayub and M. Tahir. 2014. Effect of weed control practices and irrigation levels on weeds and yield of wheat. Indian J. Agron., 52(1): 6063.

Pataczek, L., Z.A. Zahir, M. Ahmad, S. Rani, R. Nair, R. Schafleitner, G. Cadisch and T. Hilger. 2018. Beans with Benefits-The Role of Mungbean (Vigna radiata) in a Changing Environment. Am. J. Plant Sci., (9): 1577-1600.

Raza, M.H., G.U. Sadozai, M.S. Baloch, E.A. Khan, I. Din and K. Wasim. 2012. Effect of irrigation levels on growth and yield of mungbean. Pak. J. Nutr., 11(10): 974-977.

Sangakara, U.R., 1994. Yield and seed quality of mungbean as affected by irrigation in a dry season. J. Agron. Crop. Sci., 172: 327-332.
Shafique, S.,A. Javaid and S. Shafique. 2013. Management of littleseed canarygrass (Phalaris minor Retz.)by extracts and dry leaf biomass of Parthenium hysterophorus (L.). Philipp. Agric. Sci., 96(4): 426-431.

Sharma, S.N. and K.K. Singh. 2011. Seed rate and weed management on yield and nutrient uptake of wheat. Indian J. Agric. Sci., (81): 1174-1179.

Siddiqui I, R. Bajwa, Z. Huma and A. Javaid. 2010. Effect of six problematic weeds on growth and yield of wheat. Pak. J. Bot. 42(4): 2461-2471.

Tesio, F., and A. Ferrero. 2010. Allelopathy, a chance for sustainable weed management. Int. J. Sustain. Dev. World. Ecol., 17(5): 377-389.

Towa J.J. and G. Xiangping. 2014. Effects of irrigation and weed-control methods on growth of weed and rice. Int. J. Agric. Biol. Eng., 7(5): 22-33.

Yarnia, M., M.B.K. Benam and E.F.M. Tabrizi. 2010. Allelopathic effects of sorghum extracts on Amaranthus retroflexus seed germination and growth. J. Food Agric. Environ., 7 (3\&4): 770-774.

Zimdahl, R.I., 2013. Fundamentals of weed science, fourth edition. Acdemic Press. 